\title{
Non-thermal broadening of coronal emission lines in the onset phase of solar flares and CMEs
}

\author{
H. R. M. Kay, S. A. Matthews, L. K. Harra, and J. L. Culhane
}

Mullard Space Science Laboratory, University College London, Holmbury St. Mary, Dorking, Surrey, RH5 6NT, UK

e-mail: hrm@mssl.ucl.ac.uk

Received 14 April 2005 / Accepted 3 August 2005

\section{ABSTRACT}

The non-thermal broadening of soft X-ray emission lines is commonly seen during the early stages of solar flares and is thought to be associated with either the initial flare energy release or the evaporation of chromospheric plasma. Here we investigate the magnitude of non-thermal broadening for a sample of 12 flares associated with both eruptive and non-eruptive events, i.e. those with and without associated coronal mass ejections (CMEs), using the Bragg Crystal Spectrometer (BCS) on the Yohkoh spacecraft. The maximum non-thermal broadening of the eruptive flares was found to be on average lower than for the flares which were not associated with CMEs. There was no evidence of any relationship between the maximum non-thermal broadening and the initial CME speed.

Key words. Sun: activity - Sun: flares - Sun: coronal mass ejections (CMEs) - Sun: X-rays, gamma rays

\section{Introduction}

Excess broadening of soft X-ray spectral lines is commonly seen at the onset of solar flares (Antonucci et al. 1982; Fludra et al. 1989; Harra-Murnion et al. 1997). This non-thermal broadening is defined as the difference between the Doppler temperature $\left(T_{\mathrm{D}}\right)$, determined from the total line width, and the plasma temperature $\left(T_{\mathrm{e}}\right)$ calculated from line ratio diagnostics. It can also be expressed as a non-thermal velocity given by

$V_{\mathrm{nt}}=\left[2 k\left(T_{\mathrm{D}}-T_{\mathrm{e}}\right) / m_{\mathrm{i}}\right]^{1 / 2}$

where $m_{\mathrm{i}}$ is the mass of the ion considered, $k$ is the Boltzmann constant and $T_{\mathrm{e}}$ is assumed to be equal to the ion temperature $\left(T_{\mathrm{i}}\right)$. The value of $V_{\mathrm{nt}}$ typically increases during the onset of a solar flare, decreases during the impulsive phase and reaches a minimum as the soft X-ray flux nears maximum (Antonucci et al. 1982; Antonucci et al. 1984; Antonucci 1989).

The origin of the non-thermal broadening seen at the onset of solar flares is still a topic of debate. It has been suggested that it is either produced by turbulent plasma related to the initial energy release or to the motion of plasma evaporated following the injection of energy to the chromosphere. Standard models of solar flares, for example the chromospheric evaporation model of Yokoyama \& Shibata (1998), suggest that the magnetic reconnection in the flare takes place above the soft $\mathrm{X}$-ray loop tops and produces reconnection jets extending upward and downward from the reconnection X-point. Tsuneta (1995) suggests that turbulence produced by this reconnection outflow at the loop tops produces the non-thermal broadening. A second mechanism explaining how non-thermal broadening can be related to the initial energy release has been suggested by Jakimiec et al. (1998). In this scenario, the bright loop-top kernels seen in Yohkoh-SXT images, contain tangled magnetic fields. It is the MHD turbulence within these kernels that Jakimiec suggests is the cause of the non-thermal broadening. The competing scenario for the production of non-thermal broadening is directly related to the evaporation of chromospheric material during a flare. Here the line broadening is produced by the superposition of emission from upflowing material with a range of velocities and originating in either one loop or from many different loops.

In fact, a study by Ranns et al. (2001) suggests that neither mechanism may be solely responsible for the broadening, but the mechanism which dominates for any individual flare may be determined by the way in which the flare evolves. Ranns et al. (2001) examined both the magnitude and timing of the $V_{\text {nt }}$ seen in 59 solar limb flares in comparison with their hard X-ray emission, observed by the Burst and Transient Source Experiment (BATSE). They found that in the case of flares which exhibited a gradual rise in HXT emission, the $V_{\mathrm{nt}}$ peaked before the hard X-ray peak, suggesting that the nonthermal broadening is associated with the initial energy release. In the case of flares with an impulsive hard X-ray rise phase, motion due to evaporating plasma seemed to be responsible for the non-thermal broadening as the $V_{\text {nt }}$ peaked after the hard $\mathrm{X}$-ray emission. They also report tentative evidence that the levels of $V_{\mathrm{nt}}$ seen in the impulsive rise flares were larger than in the case of the gradual rise flares.

Solar flares are often accompanied by other transient events such as coronal mass ejections (CMEs), with high intensity 
and long duration flares tending to have a higher probability of being associated with a CME (Harrison 1995; Sheeley et al. 1983). Kay et al. (2003) previously studied the flare-CME relationship by examining the soft X-ray properties of flares both with and without associated CMEs. Investigation of the peak temperatures and emission measures indicated that flares which were associated with CMEs tended, on average, to reach lower peak temperatures than non-eruptive events of the same intensity. This suggests that the occurrence of a CME affects the heating and energy release in the associated flare.

In fact it has also recently been suggested that there are actually two classes of CMEs, characterised by their speed (Sheeley et al. 1999; St. Cyr et al. 1999). Slow CMEs are defined as having apparent speeds $<600 \mathrm{~km} \mathrm{~s}^{-1}$, with fast CMEs having speeds $>750 \mathrm{~km} \mathrm{~s}^{-1}$. Low \& Zhang (2002) propose a model to explain both these fast and slow CMEs, where reconnection is crucial in the early development of fast CMEs, but is not crucial (but can occur) in the case of slow CMEs. Zhang \& Golub (2003) have subsequently shown that the morphologies of the flares associated with the two types of CMEs do show marked differences.

If non-thermal broadening is related to the flare energy release and given the results of Ranns et al. (2001), we may see differences in the levels of non-thermal broadening between eruptive and compact flares. In this paper we investigate the levels of non-thermal broadening seen in flares both with and without accompanying CMEs, in an effort to probe the energy release mechanisms.

\section{Observations and analysis}

The analysis for this study falls into two main parts, i) determination as to whether the event is eruptive (i.e. is there a CME associated with the event) and ii) measurement of the nonthermal velocity. Identification of associated CMEs was done using data from the Large Angle Spectroscopic Coronagraph (LASCO: Brueckner et al. 1995) and the Extreme-ultraviolet Imaging Telescope (EIT: Delaboudinière et al. 1995) on the SOHO spacecraft. The non-thermal velocities were measured using spectral data from the Bragg Crystal Spectrometer (BCS: Culhane et al. 1991) on the Yohkoh spacecraft.

\subsection{Flare selection}

The flares were selected on the basis of the following criteria:

- there was good coverage with BCS of the rise and peak of the non-thermal broadening seen during the flare.

- the BCS spectra were clean, with no contamination from other events occurring on the solar disk at the time of maximum non-thermal broadening.

- there were sufficient LASCO and EIT data available to confirm, with a good degree of certainty, whether or not a CME was associated with the event and that there were no other flares occurring simultaneously at other locations on the solar surface.

- the flare light curve, as observed with the X-ray Sensor (XRS) on the Geostationary Operational Environmental
Table 1. The selected flares and the measured non-thermal broadening.

\begin{tabular}{lcccc}
\hline \hline Event Date & $\begin{array}{c}\text { Start time } \\
\text { (UTC) }\end{array}$ & $\begin{array}{c}\text { GOES } \\
\text { Class }\end{array}$ & Eruptive $^{a}$ & $\begin{array}{c}\text { Max } V_{\mathrm{nt}} \\
\left(\mathrm{km} \mathrm{s}^{-1}\right)\end{array}$ \\
\hline 15 Jan. 1998 & $14: 27$ & $\mathrm{M}$ & yes & $142.9 \pm 3.0$ \\
08 May 1998 & $01: 47$ & $\mathrm{M}$ & yes & $107.9 \pm 1.3$ \\
20 Feb. 2000 & $21: 58$ & $\mathrm{M}$ & yes & $>150.2 \pm 1.3$ \\
01 May 2000 & $10: 18$ & $\mathrm{M}$ & yes & $141.9 \pm 3.0$ \\
18 May 2000 & $15: 55$ & $\mathrm{M}$ & yes & $130.1 \pm 3.3$ \\
23 Jun. 2000 & $14: 13$ & $\mathrm{M}$ & yes & $123.4 \pm 3.9$ \\
\hline 17 Feb. 1998 & $09: 45$ & $\mathrm{C}$ & no & $129.5 \pm 3.2$ \\
11 Nov. 1998 & $04: 02$ & $\mathrm{M}$ & no & $167.7 \pm 2.2$ \\
20 Jun. 1999 & $08: 32$ & $\mathrm{C}$ & no & $164.3 \pm 3.4$ \\
10 Mar. 2000 & 21:01 & $\mathrm{C}$ & no & $175.3 \pm 1.1$ \\
01 Jun. 2000 & $06: 07$ & $\mathrm{M}$ & no & $181.1 \pm 1.9$ \\
27 Oct. 2000 & 20:38 & $\mathrm{M}$ & no & $157.8 \pm 3.0$ \\
\hline
\end{tabular}

${ }^{a}$ Defined in terms of whether a CME occurred with the flare.

${ }^{b}$ See the discussion of this event in Sect. 3 .

Satellite (GOES), showed a relatively simple, preferably single-peaked, event. This reduces the likelihood of choosing an event which occurs simultaneously with another event elsewhere on the disk and which could cause additional broadening in the BCS spectra.

Restricting the choice of events using the above criteria made it difficult to obtain a large number of events for our study. However, as the BCS is a full-disk spectrometer, it is necessary to ensure that the emission we are detecting is due only to the event we are considering and is not contaminated by other events occurring elsewhere on the disk. In the course of the event selection we identified and rejected over one hundred flares observed with BCS. On the basis of the selection criteria, 12 suitable events have been identified, six of which were associated with CMEs and six of which were not. The selected flares are listed in Table 1 along with the flare start time, which was determined from the GOES light curve.

\subsection{BCS observations}

The high-resolution spectra obtained by the BCS on the Yohkoh spacecraft allow the determination of the non-thermal broadening during the onset and impulsive phases of solar flares. The BCS consists of four bent-crystal spectrometers, covering the emission-line complexes of He-like S XV, Ca XIX, Fe XXV and H-like Fe XXVI. Wavelength and intensity calibration of BCS was performed at one wavelength in each of the four BCS channels. Wavelength calibration, transferable through each wavelength range from crystal curvature measurement, has a precision of between $\pm 1 \mathrm{~m} \AA$ and $\pm 5 \mathrm{~m} \AA$ depending on the individual crystal. Intensity calibration is to $\pm 15 \%$ (Culhane et al. 1991). 


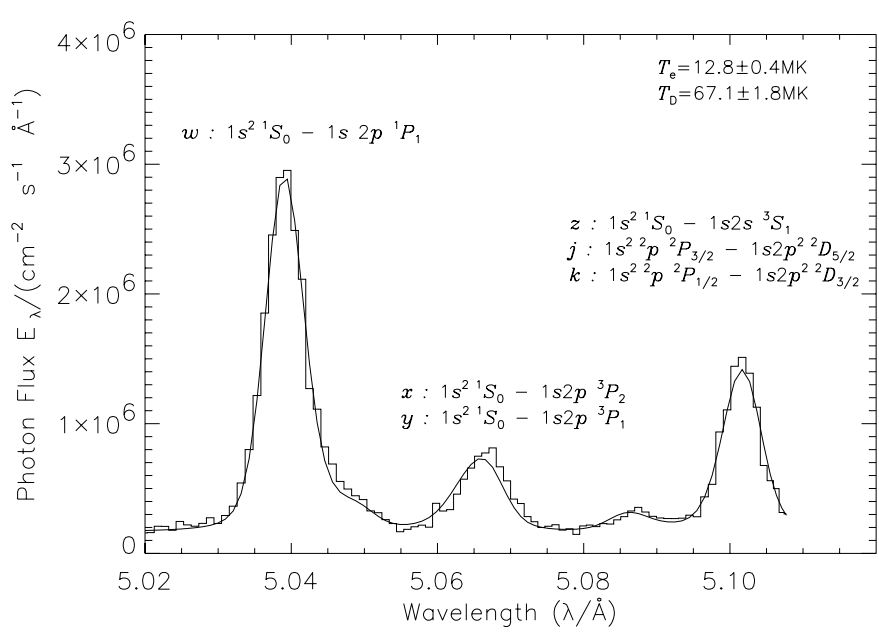

Fig. 1. An example of the spectral fit to the BCS spectrum for the flare on 11 November 1998. The components of the He-like S XV line complex, including line blends, are labelled both with their spectroscopic identification and according to the nomenclature of Gabriel (1972).

The observed non-thermal broadening for the sample of flares considered in this paper was determined using the He-like S XV emission lines, the theory of which has been detailed by Gabriel (1972) and Gabriel \& Jordan (1969). The relative intensities of the resonance line $(w)$, the intercombination line $(x+y)$, and the forbidden line $(z)$ can be used to determine both the electron temperature and density of the emitting plasma. The intensity ratio $R$, defined as the ratio of the intensities of the forbidden line to the intercombination line $I_{z} /\left(I_{x}+I_{y}\right)$, is sensitive to the electron density. Both $R$ and the intensity ratio $G$, defined as $\left(I_{x}+I_{y}+I_{z}\right) / I_{w}$, are sensitive to temperature (Gabriel \& Jordan 1969). Bely-Dubau et al. (1982) outline several techniques which can be used to apply the emission-line diagnostics of He-like atoms to the determination of plasma parameters in observed spectra.

The spectral fitting for this paper was done using standard BCS processing and analysis codes within Solarsoft (fit_bsc written by Zarro and Lemen). Spectra were accumulated until a total of 12000 counts was obtained, and a synthetic spectrum was then generated by assuming initial guesses for $T_{\mathrm{e}}, T_{\mathrm{D}}$ and the emission measure. Details of both the spectral synthesis code and the atomic data used can be found in Harra-Murnion et al. (1996). The values of these parameters were then iterated using a $\chi^{2}$ minimisation technique, until a best fit synthetic spectrum was found which reproduced the observed line flux ratios. The corresponding non-thermal velocity was then calculated from the derived values of $T_{\mathrm{e}}$ and $T_{\mathrm{D}}$. Associated uncertainties in the values of $V_{\text {nt }}$ were calculated from a Taylor series expansion of the fitted parameters. An example showing the observed spectrum at the time of maximum $V_{\text {nt }}$ for the flare which occurred on 11 November 1998, along with the best fit model, can be seen in Fig. 1. Both the spectroscopic notation and the line identifications according to the nomenclature of Gabriel (1972) are given, along with the derived values of $T_{\mathrm{e}}$ and $T_{\mathrm{D}}$.

\subsection{Detection of a $C M E$}

The presence, or lack of, a CME was determined using data from LASCO and EIT on the SOHO spacecraft. The LASCO CME catalogue of Yashiro (http: //cdaw.gsfc . nasa.gov/ CME-list/) was used to identify a CME originating from the same source region as the flare and with an onset time within one hour of the flare. This provides an initial method for associating a CME with a particular flare but is complicated by both projection effects and pinpointing the exact location of the CME source region. Mass ejection can also be seen in EIT difference images where it is characterised by large-scale dimming due to the decrease in density and hence reduced emission measure resulting from mass ejection.

EIT data, taken with the $195 \AA$ filter, were processed and calibrated using standard tasks within Solarsoft, which included flat-fielding, de-gridding and normalisation. Running difference images were then used to identify regions of dimming associated with the CMEs observed in the LASCO data, allowing the source region to be determined and providing a means of associating flares and CMEs. Dimming was seen in the eruptive events, with the exception of the flares on 1 May 2000 and 15 January 1998. No dimming was seen for any of the non-eruptive events.

In the case of the 1 May 2000 event, the associated CME has a very large initial speed $\left(1373 \mathrm{~km} \mathrm{~s}^{-1}\right)$ as determined from the time-height measurements in the CME catalogue. Given this high speed and the cadence of the EIT images (images were taken at 10:00, 10:24 and 10:36 UTC), it is possible that the dimming would have been short-lived and not visible in the EIT data. In the case of the flare on 15 January 1998, the EIT images showed little dimming during the course of the flare. The LASCO data show evidence of a CME occurring with an onset time of 14:48 UTC, i.e. during the time of the flare, and we therefore conclude that it is associated with this flare. It is possible that the CME may be due to some other event such as a filament eruption occurring independently of the flare. The daily EIT images taken with the $304 \AA$ filter do not show any evidence of any disappearing filaments between 14 and 16 January 1998, however we have not been able to obtain any $\mathrm{H}-\alpha$ data to confirm or deny this.

For those events with associated CMEs, the CME onset time was taken from the catalogue of Yashiro where it was determined from second order fits to the CME time-height data, assuming constant acceleration. These are given in Table 2 .

\section{Results and discussion}

\subsection{Maximum $V_{\mathrm{nt}}$}

Table 1 gives the date and start time of the flares considered, the GOES classification and whether or not a CME occurred in conjunction with the flare, as determined from the EIT and LASCO data. The maximum non-thermal velocity attained in each flare, from spectral fitting of the BCS data, is also listed.

Rate-dependent image distortions have been observed in one-dimensional, position-sensitive proportional counters such as those used in the BCS, where the positional linearity is affected by the intensity distribution of the incident radiation 
Table 2. Properties of the CMEs associated with the eruptive flares.

\begin{tabular}{lccccc}
\hline \hline Event date & $\begin{array}{c}\text { Start time } \\
\text { (UTC) }\end{array}$ & $\begin{array}{c}\text { CME onset }^{a} \\
\text { time (UTC) }\end{array}$ & $\begin{array}{c}\text { Quality }^{a} \\
\text { index }\end{array}$ & $\begin{array}{c}\text { Initial CME }^{b} \\
\text { speed }\left(\mathrm{km} \mathrm{s}^{-1}\right)\end{array}$ & $\begin{array}{c}\text { Corrected CME }^{c} \\
\text { speed }\left(\mathrm{km} \mathrm{s}^{-1}\right)\end{array}$ \\
\hline 01 May 2000 & $10: 18$ & $10: 22$ & 3 & 1373 & 1793 \\
18 May 2000 & $15: 55$ & $15: 45$ & 4 & 720 & 1398 \\
23 Jun. 2000 & $14: 13$ & $14: 11$ & 5 & 1005 & 1057 \\
20 Feb. 2000 & $21: 58$ & $21: 53$ & 2 & 242 & 242 \\
08 May 1998 & $01: 47$ & $01: 32$ & 3 & 482 & 482 \\
15 Jan. 1998 & $14: 27$ & $14: 48$ & 2 & 170 & 321 \\
\hline
\end{tabular}

${ }^{a}$ Taken from the LASCO CME catalogue.

${ }^{b}$ Derived from fits to the time-height data in the LASCO CME catalogue.

${ }^{c}$ Correction for projection effects (cf. Sect. 3.2).

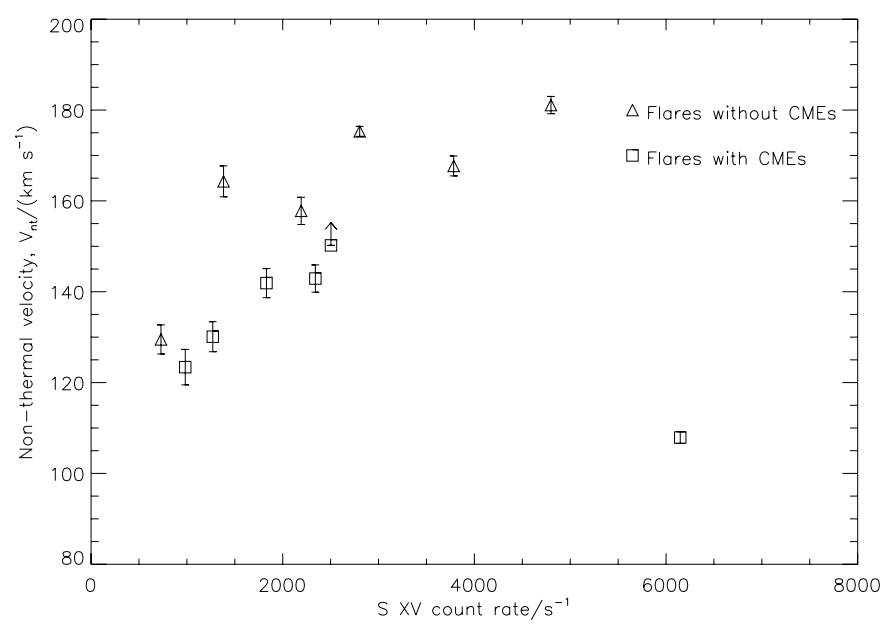

Fig. 2. The maximum $V_{\mathrm{nt}}$, and associated error, plotted against the $\mathrm{S} \mathrm{XV}$ count rate at the time when non-thermal broadening was at a maximum.

(Trow et al. 1994). This is manifested as a decrease in observed spectral line widths at high count rates. This distortion would certainly be significant at the high count rates seen in the $\mathrm{S}$ XV channel at the peak of the $\mathrm{M}$ and possibly C-class events considered in this paper. However, as we are primarily interested in the pre-flare and onset phases of the events, the lower count rates at these times will limit the impact of this instrumental line-narrowing effect.

If line narrowing were to affect significantly the nonthermal broadening observed in our sample of flares, then we would expect to see the values of maximum $V_{\text {nt }}$ decrease at higher $\mathrm{S} X \mathrm{X}$ count rates. Figure 2 shows the maximum $V_{\mathrm{nt}}$ for each of our flares along with the measured $S$ XV count rate at this time. There is no evidence for lower levels of $V_{\mathrm{nt}}$ except in the case of the flare with the highest count rate, which is the event which occurred on 18 May 2000. It may be possible therefore that the low value of maximum $V_{\mathrm{nt}}$ in this case may be affected by line narrowing. However, even if we consider this event to be suspect it does not affect the overall conclusion of this work.

The values of the maximum $V_{\text {nt }}$ seen in each of the flares have been plotted against the maximum $\mathrm{S} X V$ flare count rate in

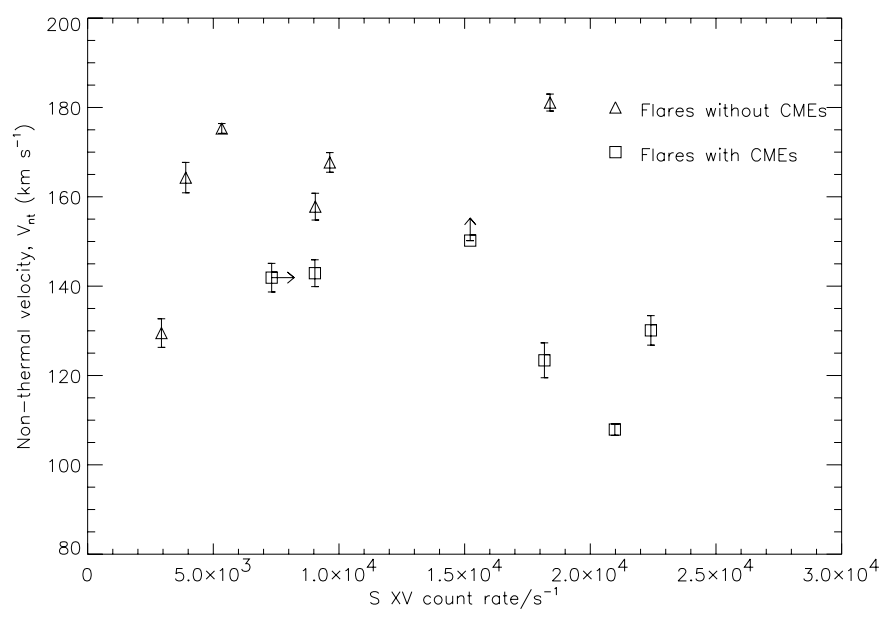

Fig. 3. The maximum $V_{\text {nt }}$, and the associated error, plotted against the peak $\mathrm{S} X V$ count rate of the flare.

Fig. 3. It is evident that the flares which occurred without associated CMEs have higher values of $V_{\mathrm{nt}}$, ranging from 129.5 to $181.1 \mathrm{~km} \mathrm{~s}^{-1}$, than those which are associated with CMEs. This suggests that for any flare of a given intensity, those without associated CMEs tend to exhibit higher levels of non-thermal broadening. The one exception in the sample is the flare which occurred on 17 February 1998 which had a maximum $V_{\text {nt }}$ of $129.5 \mathrm{~km} \mathrm{~s}^{-1}$. The EIT images showed no dimming associated with this flare. The LASCO CME catalogue reported a CME with an onset time more than one hour after the start of the flare. This onset time has been calculated for a distance of $1.76 R_{\odot}$ as a 2 nd order fit to the time-height data was unable to produce an onset time for $1 R_{\odot}$. The position angle of $285^{\circ}$ (measured in an anti-clockwise direction) seems higher than would be expected given that the flare occurred below the solar equator. The flare was therefore initially classified as not eruptive, but if it is indeed associated with this CME then the maximum $V_{\text {nt }}$ would be consistent with the other eruptive flares.

The mean value of maximum $V_{\mathrm{nt}}$ in the case of the six flares without associated CMEs is $162.6 \pm 6.8 \mathrm{~km} \mathrm{~s}^{-1}$, whilst for flares with associated CMEs the value is $132.7 \pm 5.8 \mathrm{~km} \mathrm{~s}^{-1}$, the quoted uncertainty represents the standard error on the mean. Applying a Student t-test indicates the mean values 
of $V_{\mathrm{nt}}$ for the two types of event are significantly different at the $95 \%$ level. If we categorize the event which occurred on 17 February 1998 as eruptive, then the mean value of maximum $V_{\mathrm{nt}}$ for flares without associated CMEs becomes $169.2 \pm 3.7 \mathrm{~km} \mathrm{~s}^{-1}$, whilst for flares with CMEs the value becomes $132.3 \pm 5.0 \mathrm{~km} \mathrm{~s}^{-1}$. Applying a Student t-test shows the mean values of $V_{\text {nt }}$ are significantly different at the $99 \%$ level.

As Ranns et al. (2001) point out, flares with gradual hard $\mathrm{X}$-ray emission have a tendency to be related to two-ribbon flares and long duration events (LDEs), which are commonly eruptive events and can therefore be tentatively compared to our events with associated CMEs. On the other hand, flares with impulsive hard X-ray bursts tend to be compact or confined flares. The weak evidence of higher levels of $V_{\text {nt }}$ seen in their impulsive hard X-ray events would seem therefore to be confirmed by the results presented here. It may be simply then, as Ranns et al. (2001) suggest, that different mechanisms operate in the case of eruptive and confined events, producing the different levels of maximum $V_{\mathrm{nt}}$ seen in our results. However, it is also important to consider how the different mechanisms which have been proposed to account for non-thermal broadening could produce our results.

The derived onset times for the CMEs in our sample are not sufficiently accurate to allow us to determine whether they occurred before the start of the associated flares. However, in models of CME initiation, such as the breakout model (Antiochos et al. 1999), the initial opening of magnetic field lines in the mass ejection removes the overlying unsheared field and also allows the low-lying sheared flux to open. This may reduce the complexity of the magnetic field configuration, and hence the turbulence, in the kernels suggested by Jakimiec et al. (1998). In addition, a study of the peak temperature and emission measure of both eruptive and compact flares by Kay et al. (2003) found that for a given peak soft X-ray flare intensity, eruptive flares tended on average to have lower peak temperatures. It would therefore seem plausible that energy from the active region is removed by the CME prior to the flare, resulting in less energy being available for release in the flare itself. This could result in a less intense reconnection jet and hence lower levels of turbulence being subsequently produced. However, this could also lead to a reduction in the hard X-ray emission produced in the flare, which was not seen in the study by Ranns et al. (2001). A further study of non-thermal broadening and hard X-ray emission in flares is planned for the future using data from the Ramaty High-Energy Solar Spectroscopic Imager (RHESSI) and the Solar-B observatory.

If the broadening is in fact due to the superposition of plasma flows, then in the case of eruptive flares, the associated CME would produce outflowing material where the dominant motion would be in a radial direction. This would result in a more restricted distribution of observed velocities, and hence a reduction in the maximum $V_{\mathrm{nt}}$ observed.

\subsection{CME timing and speed}

If the tendency for flares without CMEs to have higher maximum $V_{\text {nt }}$ that we see here indicates a causal relationship, then we might expect the non-thermal broadening in eruptive flares to be affected by the amount of energy released in a CME and hence the speed at which the material is ejected from the solar atmosphere. The CME catalogue of Yashiro provides a list of CMEs detected by LASCO, including information on the time-height profiles. These have been used to provide information on the projected CME speed and the estimated onset time, determined from 2 nd order fits to the time-height data. This is the time at which the CME would have been at a distance of $1 R_{\odot}$ from the solar centre. The onset times for the CMEs in our sample are listed in Table 2. The LASCO observations of each CME are assigned a quality index in the catalogue (where a higher number corresponds to a higher quality observation, and a value of three represents a typical observation) and these are also listed in Table 2. These give an indication of the reliability of the onset times and estimated CME speeds.

As can be seen, the CMEs tend to occur within \pm 21 min of the flare start times. We have estimated the initial speed of the $\mathrm{CME}$ as it left the solar surface from the first two time-height measurements in the catalogue and these are recorded in column five of Table 2. These speeds are only the projected CME speeds as measured in the plane of the sky. However, many of our CMEs and flares originate on the solar disk far from the limb and hence the projected speed is only a lower limit. By making the assumptions that the spatial origin of the CME and flare are co-incident and that the CME material leaves the Sun radially, we can use the longitudinal location of the associated flare to provide a more realistic estimate of the radial CME speed. These radial CME speeds are listed in column six of Table 2.

The relationship between the CME speed and the maximum $V_{\text {nt }}$ is shown in Fig. 4. None of the plots show any relationship between the CME speed and the maximum $V_{\mathrm{nt}}$. However, we are of course limited by the small number of events contained in our sample. We are also constrained by the errors associated with measuring the time-height profiles in the LASCO data. These include the often small number of images available and the identification of features in the CME material which can be tracked, both of which will greatly affect the determination of the CME speed. We do not however, see any indication that the maximum $V_{\mathrm{nt}}$ seen in the flare and the initial speed of the CME are related.

It is interesting to note however, that in Fig. $4 \mathrm{c}$, the three flares with CME speeds $<600 \mathrm{~km} \mathrm{~s}^{-1}$ (the threshold for fast and slow CMEs discussed by Sheeley et al. 1999 and St. Cyr et al. 1999) show a trend of decreasing $V_{\mathrm{nt}}$ with increasing CME speed, while the opposite is true for flares with CME speeds $>1000 \mathrm{~km} \mathrm{~s}^{-1}$. We are of course limited to only six events, and any apparent trend may just be due to co-incidence, and so a further study with a larger number of events would prove useful.

\section{Conclusions}

We have investigated the levels of non-thermal broadening in emission lines for a sample of C and M-class flares, using data from Yohkoh-BCS. The sample included six flares which were associated with CMEs and six which were not. 

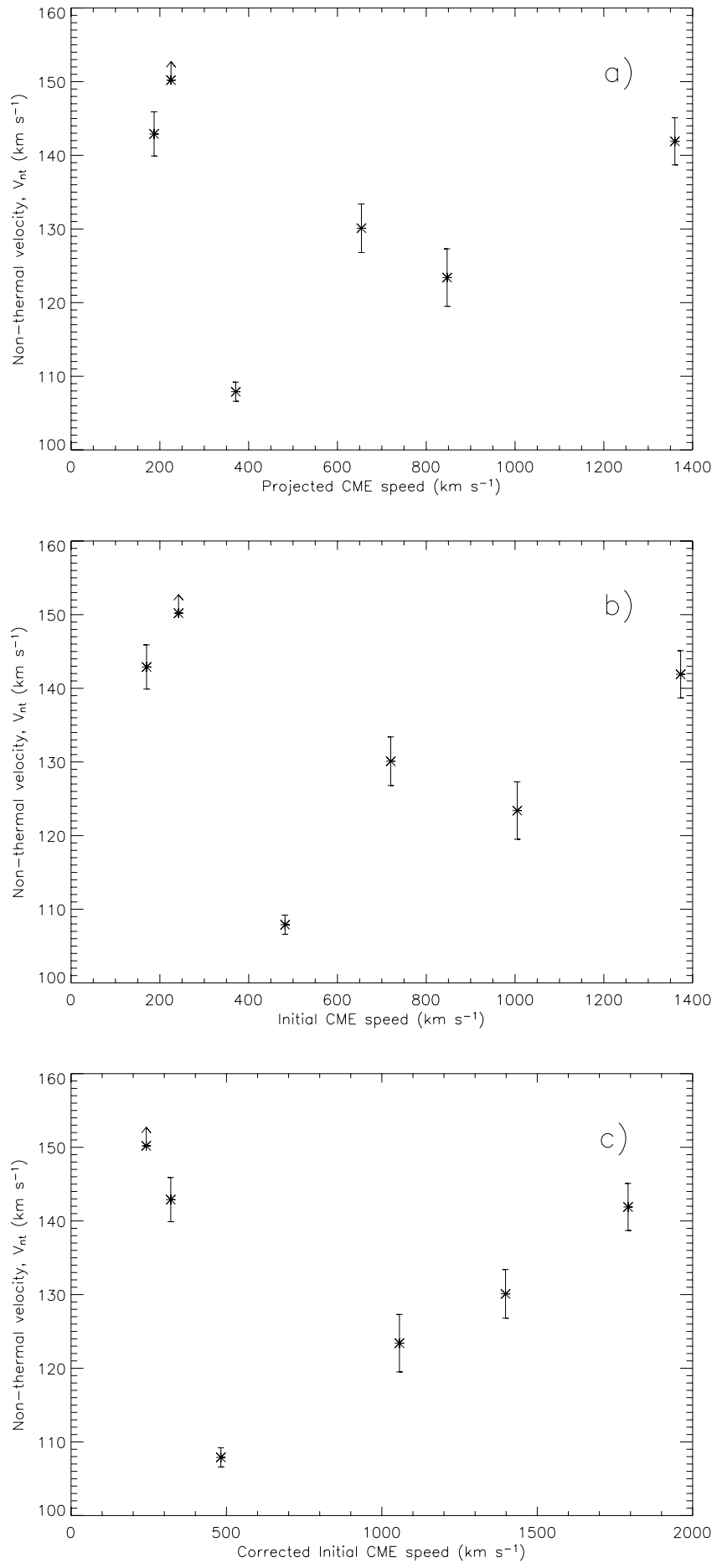

Fig. 4. The relationship between maximum non-thermal broadening and CME speed. a) the average projected CME speed, b) the initial linear speed, c) the initial linear speed corrected for the flare/CME location.

The results showed that i) the maximum non-thermal broadening seen for the eruptive flares tended to be lower than for the confined flares of the same intensity. The average maximum $V_{\mathrm{nt}}$ for compact flares was found to be $162.6 \pm 6.8 \mathrm{~km} \mathrm{~s}^{-1}$, whilst for flares with associated CMEs the value was $132.7 \pm 5.8 \mathrm{~km} \mathrm{~s}^{-1}$; ii) no conclusive relationship was found between the initial CME speed, as determined from LASCO CME data, and the maximum $V_{\mathrm{nt}}$. The differing levels of $V_{\mathrm{nt}}$ seen in the two types of event tend to support the results of Ranns et al. (2001) who suggest that the mechanism responsible for the non-thermal broadening may be dictated by the flare evolution. Our results do not allow us to discriminate whether turbulent plasma, related to the initial energy release, or evaporated chromospheric plasma is responsible in the case of our flares.

One of the drawbacks in using BCS data for this study is the difficulty in finding suitable flares. BCS observations are frequently interrupted by periods during which the Sun is not observed (e.g. Yohkoh night and passage through the South Atlantic Anomaly). It therefore becomes difficult to find observations of suitable flares, where the pre-flare, onset and decay of the flare have all been observed. This, combined with the problem of rate-dependent image distortions which restricts our analysis us to lower intensity events, results in a limited number of events that we can include in this study.

The Solar-B observatory, which will be launched in 2006 will include both an X-Ray Telescope (XRT) and a EUV Imaging Spectrometer (EIS). The advantage of both spatiallyresolved and more continuous observations which will be available with EIS will enable us to construct a much larger sample of both eruptive and non-eruptive flares, allowing us to make a larger statistical study, along with a more accurate determination of the magnitude of non-thermal broadening. Additionally, 2006 will also see the launch of the STEREO spacecraft. This will provide us with 3-D images of the solar corona via the Earth Connection Coronal and Heliospheric Investigation (SECCHI) set of instruments, enabling us to make more confident associations between individual flares and CMEs. This will also provide us with more accurate $\mathrm{CME}$ onset times and CME speeds.

Acknowledgements. H.R.M.K. acknowledges financial support from the UK Particle Physics and Astronomy Research Council (PPARC). This work used data obtained from the Solar UK Research Facility (SURF) at the University College London, Mullard Space Science Laboratory (MSSL). The SOHO/LASCO data used in this work are produced by a consortium of the Naval Research Laboratory (USA), Max-Planck-Institut für Sonnensystemforschung (Germany), Laboratoire d'Astronomie (France) and the University of Birmingham (UK). SOHO is a project of international co-operation between ESA and NASA. The Yohkoh satellite is a project of the Institute of Space and Astronautical Science. The CME catalogue of Yashiro is generated and maintained by NASA and the Catholic University of America in cooperation with the Naval Research Laboratory.

\section{References}

Antiochos, S. K., DeVore, C. R., \& Klimchuk, J. A. 1999, ApJ, 510, 485

Antonucci, E. 1989, Sol. Phys., 121, 31

Antonucci, E., Gabriel, A. H., Acton, L. W., et al. 1982, Sol. Phys., 78, 107

Antonucci, E., Gabriel, A. H., \& Dennis, B. R. 1984, ApJ, 287, 917

Bely-Dubau, F., Faucher, P., Steenman-Clark, L., et al. 1982, MNRAS, 201, 1155

Brueckner, G. E., Howard, R. A., Koomen, M. J., et al. 1995, Sol. Phys., 162, 233

Culhane, J. L., Bentley, R. D., Hiei, E., et al. 1991, Sol. Phys., 136, 89 
Delaboudinière, J.-P., Artzner, G. E., Brunaud, J., et al. 1995, Sol. Phys., 162, 291

Fludra, A., Lemen, J. R., Jakimiec, J., Bentley, R. D., \& Sylwester, J. 1989, ApJ, 344, 991

Gabriel, A. H. 1972, MNRAS, 160, 99

Gabriel, A. H., \& Jordan, C. 1969, MNRAS, 145, 241

Harra-Murnion, L. K., Phillips, K. J. H., Lemen, J. R., et al. 1996, A\&A, 308, 670

Harra-Murnion, L. K., Akita, K., \& Watanabe, T. 1997, ApJ, 479, 464 Harrison, R. A. 1995, A\&A, 304, 585

Jakimiec, J., Tomczak, M., Falewicz, R., Phillips, K. J. H., \& Fludra, A. 1998, A\&A, 334, 1112

Kay, H. R. M., Harra, L. K., Matthews, S. A., Culhane, J. L., \& Green, L. M. 2003, A\&A, 400, 779
Low, B. C., \& Zhang, M. 2002, ApJ, 564, L53

Ranns, N. D. R., Harra, L. K., Matthews, S. A., \& Culhane, J. L. 2001, A\&A, 379, 616

Sheeley, N. R. J., Howard, R. A., Koomen, M. J., \& Michels, J. 1983, ApJ, 272, 349

Sheeley, N. R., Walters, J. H., Wang, Y.-M., \& Howard, R. A. 1999, J. Geophys. Res., 104, 24739

St. Cyr, O. C., Burkepile, J. T., Hundhausen, A. J., \& Lecinski, A. R. 1999, J. Geophys. Res., 104, 12493

Trow, M. W., Bento, A. C., \& Smith, A. 1994, Nucl. Inst. Methods Phys. Res., 348, 232

Tsuneta, S. 1995, PASJ, 47, 691

Yokoyama, T., \& Shibata, K. 1998, ApJ, 494, L113

Zhang, M., \& Golub, L. 2003, ApJ, 595, 1251 